\title{
Demographic Analysis of Patients With Multiple Sclerosis; Individual Variability Related to the Time Interval
}

\author{
Zahra Tolou-Ghamari ${ }^{1, *}$ \\ ${ }^{1}$ Isfahan Neurosciences Research Centre, Faculty of Medicine, Isfahan University of Medical Sciences, Isfahan, IR Iran \\ *Corresponding author: Zahra Tolou-Ghamari, Isfahan Neurosciences Research Centre, Faculty of Medicine, Isfahan University of Medical Sciences, Isfahan, IR Iran. Tel/Fax: \\ +98-3116291050, E-mail: toloeghamari@pharm.mui.ac.ir
}

Received: July 6, 2014; Revised: November 19, 2014; Accepted: December 9, 2014

\begin{abstract}
Background: According to the published articles, there is an increase in the prevalence of MS all over the world. Regarding demographic characteristics for unknown reasons, females are more affected than males.

Objectives: The aim of this study was demographic analysis and model of changes in patients for three years in a local teaching hospital in Isfahan/Iran.

Patients and Methods: In total, 1500 patients with MS were studied within three Intervals (I) from; 1- 2011/05/22 to 2012/03/18 called I1, 2-2012/03/22 to 2013/03/19 called I2 and 3-2013/03/22 to 2013/10/19 called I3. Data was recorded in Excel and analyzed using SPSS for windows. Results: Of the total population studied, 75\% were female. 32\% and 33\% of patients related to the age groups of 20 - 30 and 30 - 40 years, respectively. However the number of patients related to time interval I3 were 472, but there was an increase in the number of patients studied from I1 to I2 (394 vs. 634). Approximately 13\% of total population had MS attack more than 4 to 19 times.

Conclusions: In agreement with previous reports, due to unknown reasons, the number of patients in general and thenumber of females in particular were increased annually. Within this population due to inter- and intra-individual variability, pharmacokinetic studies seem to be valuable.
\end{abstract}

Keywords: Multiple Sclerosis; Demography; Pulse therapy, Drug

\section{Background}

Over the recent years, increased prevalence of multiple sclerosis (MS) mainly in women has been the focus of concern in clinical neurology. MS and chronic fatigue syndrome are two widespread diseases with increased frequencies in middle aged females. MS could be categorized as the most prevalent persistent challenging syndrome of the central nervous system (CNS), which is associated with disability and many other rigorous clinical and psychological problems and even death. Somatosensory, visual, cognitive and psychiatric discrepancies may be present depending on the site and dimension of significant demyelination in the CNS.

Regarding the prevalence of MS, latest report estimated prevalence of 100000 and 400000 in the United Kingdom and the United States, respectively (1-7). Research reported that MS is not only a frequently seen in childhood and adolescence, but also genetic and ecological issues seem to be important. Also there is evidence for an association between BMI and MS in the second decade of life. BMI association with MS is as large as other known MS risk features (8). Research shows that cigarette smoking and helminthic infection increase the prevalence of inflammatory disease and may exaggerate cognition in patients with MS (7, 9). Brain lesion localization in MS is thought to have a role. It seems that there is a higher role for injuries close to brain ventricles. There is not sufficient data to support such a hypothesis that having a lesion close to the ventricle versus elsewhere in the brain might be more associated with unfavorable effects (10). Patients with MS have many clinical presentations such as osteoporosis, sleep disorders and many other complications (11, 12). Recent publications confirm that the incidence and prevalence of MS in Isfahan province of Iran has been increasing rapidly, especially in females (13). Like other autoimmune diseases, for unidentified reasons, there is an elevated female prevalence. Therefore, demographic analysis of patients with MS and model of changes between the time intervals were of interest.

\section{Objectives}

As the number of patients with MS increases annually in Isfahan/Iran, the purpose of this study was to examine demographic characteristics and the model of changes within a defined time interval in a local teaching hospital. 


\section{Patients and Methods}

From May 22, 2011 to October 19, 2013, patients with MS $(n=1500)$ comprised of 1119 females and 381 males were studied based on a cross-sectional method. The patients attended MS neurology clinic in 2970 events. Regarding the time interval, three divisions were made; 1 - time in terval one or I1 from $2011 / 05 / 22$ to $2012 / 03 / 18$, 2- time interval two or I2 from 2012/03/22 to 2013/03/19 and 3- time interval three or I3 from 2013/03/22 to 2013/10/19. Name, surname, age, father's name, date of attending neurology clinic and patient's hospital record number were recorded in d-Base. Statistical analyses of d-Base were performed using Microsoft Excel and SPSS (Version 18) for windows.

\section{Results}

As shown in Table 1, time intervals expressed as days, which could be described as I1 (298 days), I2 (375 days) and I3 (295 days). The total number of patients that could be referred during that period were as follows; 394 (I1; 309 females vs. 85 males), 634 (I2; 472 females vs. 162 males) and 472 (I3; 338 females vs. 134 males). Figure 1 shows the distribution of age in total population of patients studied. Among total population studied, the highest (87\%) and lowest percentage (9\%) of patients were at ages 20 - 50 and 50 - 90 years, respectively. Patients with age between 20-30, 30-40 and 40-50 years included 32\%, $33 \%$ and $22 \%$. $6 \%$ of the total population studied aged 50 to 60 years.

Females constituted 78\% (I1), 74\% (I2) and 72\% (I3) of patients. During intervals I1, 394 patients with MS visited neurology clinic on 779 events. As shown in Figure 2 , although $57 \%$ of total population studied within I1, visited neurology clinic just for once, $18 \%$ of those visited neurology clinic more than 4 up to 19 times. During interval I2, 634 patients visited neurology clinic in 1265 events. Of these populations, 379 patients visited neurology clinic just for once. During the time interval I3, 472 patients visited neurology clinic in 926 events. Of these patients, 339 individuals visited neurology clinic just for once.

During the time intervals I 2 and I3, $14 \%$ and $8 \%$ of patients attended neurology clinic for 4 to 15 times. The situations related to patients with several MS attacks could be discussed in many cases in this study, as individual cases. For example, patient with code no 1493 was a 29-year-old female that visited MS clinic 15 times during 712 days. As shown in Figure 3, she visited neurology clinic in 14 June 2011. Due to MS attack, she was eligible for MPpulse therapy. To date 28 May 2013, as it could be seen in Figure 2, with a gap of $25,41,218,30,24,36,23,47,88,45$, $28,30,33$ and finally 43 days she visited neurology clinic for pulse therapy. A 24-year-old male and a 27-year-old female with code numbers 555 and 608 visited MS clinic for pulse therapy 12 times during approximately 500 and 600 days, respectively.

\begin{tabular}{lcccc}
\hline \multicolumn{5}{l}{ Table 1. Demographic Information Between the Time Intervals } \\
\hline Patients & Total Period & I1 (298, d) & I2 (375, d) & I3 (295, d) \\
\hline Total & 1500 & 394 & 634 & 472 \\
Females & 1119 & 309 & 472 & 338 \\
Males & 381 & 85 & 162 & 134 \\
\hline
\end{tabular}

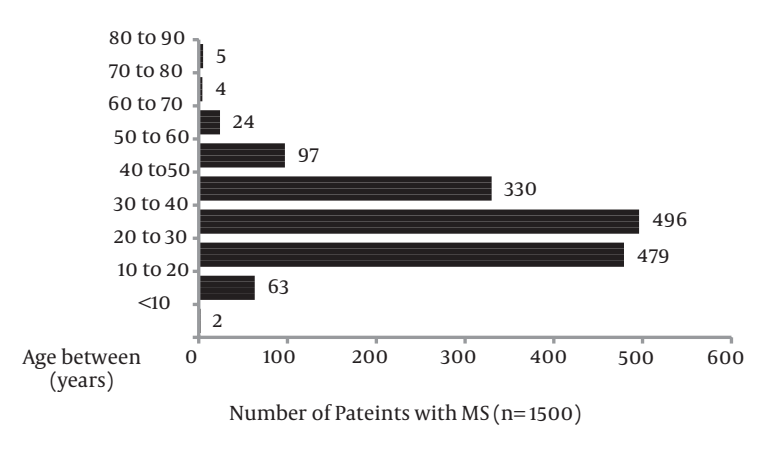

Figure 1. Distribution of Age in Patients Studied $(n=1500)$

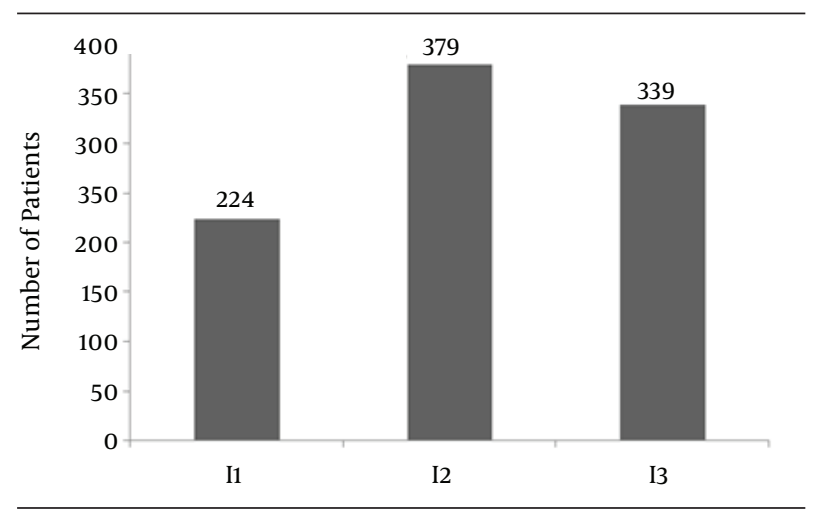

Figure 2. The Number of Patients With no MS Attacks on Time Intervals Studied $(n=1500)$

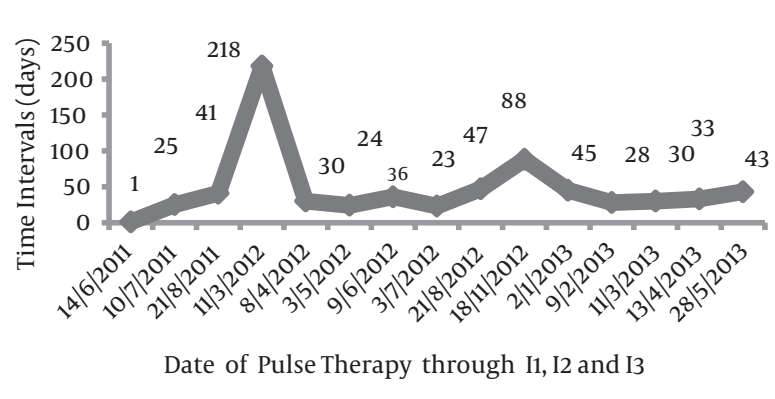

Figure 3. The Pattern of Methylprednisolone Pulse Therapy in a 24-YearOld Female.

\section{Discussion}

In agreement with previous evaluations, this investigation also supported that the highest rate of patients 
belonged to the age group 20 - 50 years old (87\%) (5) . According to the published reports, there is a medium to high risk related to the prevalence of MS in Isfahan/Iran (13). Although the pattern of changes in number of patients could be categorized as I1; 394, I2; 634 and I3; 472, the number of females overweighed males (I1; 309 vs. 85, I2; 472 vs. 162 and I3; 338 vs. 134). Publications also confirm an increased incidence of MS primarily in women over the recent years $(1,5,13)$. Due to anti-inflammatory and immunosuppressant effects of glucocorticoid, many studies recommended a well-defined border related to its use in MS by an expert neurologist. The high-dose intravenous methylprednisolone for the first three days followed by an 11-day course of low-dose oral prednisone is recommended (14). Regarding patients' demand for pulse therapy of more than four times, the model could be illustrated as; I; $18 \%$ vs. I2; $14 \%$ vs. I3; $8 \%$. Related to prognostic marker for MS, it is well known that cortical atrophy is already present at the time of MS onset (15). Studies suggest that IL2A polymorphism might be associated with disease attacks (16-19). Another study proposed that switch-associated protein 70 antibodies could be involved in MS relapse (20). Recovery from acute optic neuritis seems to be associated with vitamin D levels in patients with MS $(21,22)$. In demyelination, generation of ATP might be changed, as oligodendrocytes pass lactate to myelinated axons as a metabolic substrate for mitochondria. Furthermore, mitochondrial abnormalities inside neuronal cell bodies in those experiencing MS attacks could lead to mitochondrial respiratory sequence complex or enzyme discrepancy (23). As a final conclusion, the present study focused on demographic analysis of patients with MS, but additional research on various roles of antibodies and disease modifying drugs related to clinical pharmacokinetics aspects seem to be necessary for individualized pharmacotherapy outcome prediction.

\section{Acknowledgements}

We are thankful to Isfahan Neurosciences Research Center; INRC for their support.

\section{Funding/Support}

Isfahan University of Medical Sciences supported this study.

\section{References}

1. Laks J, Ahlgren C, Oden A, Lycke J. High Nationwide Incidence of Multiple Sclerosis in Sweden. PLOS ONE. 2014;9(9):e108-599.

2. Methley AM, Chew-Graham C, Campbell S, Cheraghi-Sohi S. Experiences of UK health-care services for people with Multiple Sclerosis: a systematic narrative review. Health Expect. 2014:12-3.

3. Tullman MJ. Overview of the epidemiology, diagnosis, and disease progression associated with multiple sclerosis. Am J Manag Care. 2013;19(2 Suppl):S15-20.
4. Evans C, Beland S, Kulaga S, Wolfson C, Kingwell E, Marriott J, et al. Incidence and Prevalence of Multiple Sclerosis in the Americas: A Systematic. Neuroepidemiology. 2013;40:195-210.

5. Tolou-Ghamari Z, Shaygannejad V, Ashtari F. Preliminary study related the incidence of methylprednisolone pulse therapy in patients visited multiple sclerosis clinic located at the isfahan kashani hospital. Int J Prev Med. 2013;4(Suppl 2):S274-8.

6. Ozcan ME, Ince B, Bingol A, Erturk S, Altinoz MA, Karadeli HH, et al. Association between smoking and cognitive impairment in multiple sclerosis. Neuropsychiatr Dis Treat. 2014;10:1715-9.

7. Gaber TA, Oo WW, Ringrose H. Multiple Sclerosis/Chronic Fatigue Syndrome overlap: When two common disorders collide. NeuroRehabilitation. 2014;35(3):529-34.

8. Gianfrancesco MA, Acuna B, Shen L, Briggs FBS, Quach H, Bellesis $\mathrm{KH}$, et al. Obesity during childhood and adolescence increases susceptibility to multiple sclerosis after accounting for established genetic and environmental risk factors. Obesity Res Clin Pract . 2014;8(5):e435-47.

9. Weinstock JV, Elliott DE. Helminth infections decrease host susceptibility to immune-mediated diseases. I Immunol. 2014;193(7):3239-47.

10. Friede T, Eloyan A, Shou H, Shinohara RT, Sweeney EM, Nebel MB, et al. Health Effects of Lesion Localization in Multiple Sclerosis: Spatial Registration and Confounding Adjustment. PLOS ONE. 2014;9(9):e107-263.

11. Vitkova M, Gdovinova Z, Rosenberger J, Szilasiova J, Nagyova I, Mikula P, et al. Factors associated with poor sleep quality in patients with multiple sclerosis differ by disease duration. Disability Health J. 2014;7(4):466-71.

12. Roos PM. Osteoporosis in neurodegeneration. J Trace Elements Med Biol. 2014;28(4):418-21.

13. Etemadifar M, Sajjadi S, Nasr Z, Firoozeei TS, Abtahi SH, Akbari M, et al. Epidemiology of multiple sclerosis in Iran: a systematic review. Eur Neurol. 2013;70(5-6):356-63.

14. Goodin DS. Glucocorticoid treatment of multiple sclerosis. Handb Clin Neurol. 2014;122:455-64.

15. Fernandez-Jaen A, Fernández-Mayoralas DM, Fernández-Perrone AL, Jimenez de la Pena M, Recio Rodriguez M, Calleja-Perez B, et al. Cortical thickness at the time of the initial attack in two patients with paediatric relapsing-remitting multiple sclerosis. Eur J Paediatr Neurol. 2014;18(3):295-300.

16. Ramagopalan SV, Mowry EM, Carey RF, Blasco MR, Pelletier J, Duquette P, et al. Association of Multiple Sclerosis Susceptibility Variants and Early Attack Location in the CNS. PLOS ONE. 2013;8(10):e755-65.

17. Citro A, Barnaba V, Martini H. From T Cell Apoptosis to Chronic Immune Activation in Inflammatory Diseases. Int Arch Allergy Immunol . 2014;164(2):140-6.

18. Zahednasab H, Balood M, Harirchian MH, Mesbah-Namin SA, Rahimian N, Siroos B. Increased autotaxin activity in multiple sclerosis. J Neuroimmunology. 2014;273(1-2):120-3.

19. Bsibsi M, Peferoen LAN, Holtman IR, Nacken PJ, Gerritsen WH, Witte ME, et al. Demyelination during multiple sclerosis is associated with combined activation of microglia/macrophages by IFN-y and alpha B-crystallin. Acta Neuropathologica. 2014;128(2):215-29.

20. Turkoglu R, Gencer M, Ekmekci D, Ulusoy C, Erdag E, Sehitoglu E, et al. Switch-Associated Protein 70 Antibodies in Multiple Sclerosis: Possible Association with Disease Progression. Med Principles Pract. 2014;23(3):239-45.

21. MalikMT, Healy BC, Benson LA, Kivisakk P, Musallam A, Weiner HL, et al. Factors associated with recovery from acute optic neuritis in patients with multiple sclerosis. Neurology. 2014;82(24):2173-9.

22. Shaygannejad V, Tolou-Ghamari Z. What is the Real Fate of Vitamin D in Multiple Sclerosis? Int J Prev Med. 2013;4(Suppl 2):S159-64.

23. Campbell GR, Worrall JT, Mahad DJ. The central role of mitochondria in axonal degeneration in multiple sclerosis. Mult Scler. 2014;20(14):1806-13. 